\title{
PLATAFORMA ARDUINO APLICADA NO DESENVOLVIMENTO DE UM SISTEMA DE COLETA DE DADOS MICRO-AMBIENTAIS EM CASAS DE VEGETAÇÃO
}

\author{
ARDUINO PLATFORM APPLIED IN THE DEVELOPMENT OF A \\ MICROENVIRONMENTAL DATA COLLECTION SYSTEM IN GREENHOUSES \& \\ PLATAFORMA ARDUINO APLICADA EN EL DESARROLLO DE UN SISTEMA DE \\ ADQUISICIÓN DE DATOS MICROAMBIENTALES EN INVERNADEROS \&
}

Recebido em: 20/06/2021 - Aprovado em: 12/08/2021 - Publicado em: 31/08/2021

d.

http://dx.doi.org/10.18011/bioeng2021v15n2p190-206

Aldir Carpes Marques Filho' (aldir.marques@unesp.br)

Jean Paulo Rodrigues² (jeanpaulo@ifsc.edu.br)

Gabriel Buligon Dal Ponte ${ }^{2}$ (gabriel.dalponte@hotmail.com)

\footnotetext{
1 Universidade Estadual Paulista. FCA - Botucatu, SP, Brasil.

2 Instituto Federal de Santa Catarina. IFSC - Florianópolis, SC, Brasil.
}

\section{RESUMO}

A coleta de dados e o monitoramento ambiental na agricultura são atividades de alta complexidade, demandando normalmente equipamentos de alto custo. Objetivou-se desenvolver um sistema de coleta de dados, de baixo custo, para a obtenção de parâmetros relacionados à luminosidade, umidade do solo, umidade do ar e temperatura em ambiente agrícola. Utilizou-se a plataforma de prototipagem Arduino para montagem do sistema Datalogger. Foram realizados testes de funcionamento em duas casas de vegetação A1 e A2, pelo período de 24 horas. A concepção do sistema, com a utilização de suportes de sensores, permitiu a coleta de dados sem interrupção e com adequado armazenamento na memória do dispositivo. A plataforma Arduino e os sensores acessórios, mostraram-se perfeitamente aplicáveis para a aquisição e armazenamento de dados em casas de vegetação. O protótipo de Datalogger desenvolvido apresentou redução de custo de 600 até $3000 \%$ em relação aos componentes disponíveis no mercado com funcionalidades semelhantes.

Palavras-chave: Pesquisa. Sensores. Automação. Agricultura de precisão. 


\section{INTRODUÇÃO}

A inovação nos processos agrícolas pode incrementar a produtividade das culturas e aprimorar significativamente a eficiência das lavouras (AGUDO et al. 2020). A agricultura é uma atividade que demanda a coleta de dados e informações, entretanto, a disponibilidade de equipamentos para essa função é limitada. Ali et al. (2016), afirmaram que a coleta de dados agrícolas é uma tarefa que pode ser restringida pela utilização de sistemas de alto custo, nem sempre viáveis a pequenos e médios produtores.

A informática e eletrônica têm evoluído sobremaneira no ambiente agrícola, grande parte das novas tecnologias, contam com a utilização de "microcontroladores" (NÓBREGA et al., 2020). Estes são componentes eletrônicos ou "chips", capazes de realizar algumas rotinas de programação previamente definidas, contemplando em seu hardware, processador e memória interna, além de portas para interconexão de sensores e atuadores (MCROBERTS, 2018).

Com o intuito de popularizar a aplicação dos microcontroladores surgiram as plataformas de prototipagem, que nada mais são do que os próprios microcontroladores eletrônicos, cercados por circuitos eletrônicos acessórios, que permitem a intercomunicação e interconexão amigável com sistemas externos.

Dentre as plataformas de desenvolvimento mais utilizadas no mundo está a plataforma Arduino, criada na Itália com o objetivo de popularizar a tecnologia de prototipagem (MCROBERTS, 2018). Nos últimos anos, muitos projetos são realizados com o uso desta plataforma em diversas aplicações (MEGANTORO et al., 2020; OLADIMEJI et al., 2020). As plataformas de prototipagem podem aprimorar os processos produtivos (MARTINEZ-SANTOS; ACEVEDO-PATINO e CONTRERAS-ORTIZ, 2017).

Benghanem (2009) e Baker (2014), utilizando microcontroladores em sistemas de monitoramento, ratificaram que os sistemas de baixo custo podem trazer bons resultados de implantação e funcionamento em variadas aplicações, entretanto, Gopinath et al. (2020) afirmou que para aplicações mais severas de processamento as plataformas e suas interfaces de programação apresentam limitações.

Com base no exposto acima, objetivou-se no presente trabalho, desenvolver um sistema de baixo custo para aquisição de dados micro ambientais no meio agrícola, utilizando-se componentes eletrônicos facilmente disponíveis no mercado e que possam integrar suportes específicos, instalados no estrato da cultura vegetal. 


\section{MATERIAIS E MÉTODOS}

Para desenvolver o sistema de coleta de dados, utilizou-se a metodologia de desenvolvimento de produtos proposta por Rozenfeld et al. (2015), onde foram realizados levantamentos de mercado em busca de soluções existentes e sistemas eletrônicos que atenderiam as necessidades do projeto, esta etapa foi classificada como projeto informacional. Logo em seguida ao projeto informacional, foi iniciado o projeto conceitual, onde foram levantados os requisitos do produto, definidos pelo baixo custo e pela disponibilidade de entradas digitais e analógicas.

A premissa inicial do projeto, ou o requisito básico do produto, partiu da necessidade de se desenvolver uma solução para coleta de dados ambientais com o valor máximo de duzentos dólares (200 U\$). O produto deveria ter disponibilidade mínima de 4 entradas analógicas e 5 entradas digitais para conexão de sensores diversos, como temperatura, umidade, radiação entre outros.

Inicialmente para atender os requisitos mínimos relacionado ao número de entradas e saídas, bem como, a função global do produto ser de baixo custo, foi realizada uma avaliação comparativa entre produtos disponíveis no mercado e que poderiam atender as solicitações do sistema.

Com o intuito de atender ao requisito básico do projeto no quesito "baixo custo" e a adequada disponibilidade de entradas digitais e analógicas, a plataforma Arduino, dentre outras possibilidades investigadas como CLP's (Controladores Lógicos Programáveis) e Dataloggers comerciais, foi a solução que melhor se adequou ao projeto, devido, principalmente a ampla gama de sensores e atuadores compatíveis.

O sistema de controle principal foi baseado no microcontrolador ATMEGA328P presente na plataforma "Arduino ${ }^{\mathrm{TM}}$ UNO", apresentando o total de 14 portas digitais, 6 portas analógicas, um cristal oscilador com frequência de $16 \mathrm{MHz}$, uma porta Universal Serial Bus (USB), além de uma entrada de alimentação e um botão de reset.

O sistema de aquisição de dados foi montado sobre uma placa acessória "shield" interconectada à plataforma principal de controle, contendo o relógio RTC (Real Time Clock) e o cartão de memória tipo SD (Secure Digital). A placa acessória possui fabricação pela empresa Adafruit ${ }^{\mathrm{TM}}$ Corporation. A programação do sistema foi realizada em linguagem $\mathrm{C} / \mathrm{C}++$ e foi repassada à plataforma através de software IDE Arduino ${ }^{\mathrm{TM}}$. 
A Figura 1 descreve alguns dos equipamentos com possível aplicação no projeto, e a linha de escolha das tecnologias de acordo com as características e as necessidades do produto.

Figura 1: Matriz de seleção de equipamentos para composição do projeto do sistema de monitoramento ambiental.

\begin{tabular}{|c|c|c|c|c|}
\hline FUNÇÃOO & ALTERNATIVA 1 & ALTERNATIVA 2 & ALTERNATIVA 3 & ALTERNATIVA 4 \\
\hline $\begin{array}{l}\text { CONTROLAR O } \\
\text { AMBIENTE }\end{array}$ & CLP & Microcontroladores & Arduino & Raspberry PI \\
\hline Caracteristicas Principais: & $\begin{array}{l}\text { Alto custo; Maior } \\
\text { confiabilidade e } \\
\text { funcionalidade; } \\
\text { Exige dispositivos } \\
\text { acessórios, } \\
\text { compatibilidade com } \\
\text { sensores. }\end{array}$ & $\begin{array}{l}\text { Baixo custo; Exige } \\
\text { eletrônica acessória; }\end{array}$ & $\begin{array}{l}\text { Baixo custo; } \\
\text { Eletronica acessór } \\
\text { Disponibilidade gra de } \\
\text { de sensores. }\end{array}$ & $\begin{array}{l}\text { Médio custo; } \\
\text { Eletronica acessória; } \\
\text { Memória e alto } \\
\text { processamento }\end{array}$ \\
\hline $\begin{array}{l}\text { VERIFICAR UMIDADE } \\
\text { DO SOLO }\end{array}$ & EC-5 - Decagon & 10-HS- Decagon & FC-28 & SMRT-Y \\
\hline Caracteristicas Principais: & $\begin{array}{l}\text { Alto custo; Precisão } \\
\text { de } 3 \text { a } 5 \% \text {. Garantia } \\
\text { de } 1 \text { ano. }\end{array}$ & $\begin{array}{l}\text { Alto c } \\
\text { de } 3 \% \\
\text { ano. }\end{array}$ & $\begin{array}{l}\text { Ba custo; Precisão } \\
\text { de } 5 \text { a } 10 \% \text {. Garantia } \\
\text { de } 0,25 \text { ano. }\end{array}$ & $\begin{array}{l}\text { Alto custo; Precisão } \\
\text { N.I. Garantia de } 1 \\
\text { ano. Compatib. baixa } \\
\text { microcontroladors }\end{array}$ \\
\hline $\begin{array}{l}\text { VERIFICAR UMIDADE } \\
\text { DO AR }\end{array}$ & DHT-11 & & PSICR.100 & HIGROG. \\
\hline Caracteristicas Principais: & $\begin{array}{l}\text { Baixo custo. Baixa } \\
\text { precisão. Controle } \\
\text { de umidade e } \\
\text { temperatura. }\end{array}$ & $\begin{array}{l}\text { Baixc custo. Média } \\
\text { preci ão. Controle de } \\
\text { umid de e } \\
\text { temp ratura. }\end{array}$ & $\begin{array}{l}\text { Baixo custo. Alta } \\
\text { precisão. Controla } \\
\text { somente Umidade do } \\
\text { ar. Não comunica } \\
\text { microcontrolador }\end{array}$ & $\begin{array}{l}\text { Alto custo. Alta } \\
\text { precisão. Controla } \\
\text { somente Umidade do } \\
\text { ar. Estação } \\
\text { Meteor.Analogico. }\end{array}$ \\
\hline $\begin{array}{l}\text { VERIFICAR } \\
\text { TEMPERATURA }\end{array}$ & DHT-11 & 22 & LM-35 & PT-100 \\
\hline Características Principais: & $\begin{array}{l}\text { Baixo custo. } \\
\text { precisão. Co } \\
\text { de umidadf } \\
\text { temperat' } a .\end{array}$ & $\begin{array}{l}\text { Baixo custo. Média } \\
\text { precisão. Controle de } \\
\text { umidade e } \\
\text { temperatura. }\end{array}$ & $\begin{array}{l}\text { Baixo custo. Baixa } \\
\text { precisão.Exige circuito } \\
\text { isolado. }\end{array}$ & $\begin{array}{l}\text { Médio custo. Alta } \\
\text { precisão e } \\
\text { resistência. Alto } \\
\text { Range. Ambiente } \\
\text { hostil. }\end{array}$ \\
\hline VERIFICAR RADIAÇÄO & LDK & Lux-800 & Pir.SPP & \\
\hline Características Principais: & $\begin{array}{l}\text { Baix custo. Baixa } \\
\text { prec são. Exige } \\
\text { circl to divisor de } \\
\text { tens } 0 \text {. }\end{array}$ & $\begin{array}{l}\text { Alto custo, controle } \\
\text { de luminosidade em } \\
\text { LUX. }\end{array}$ & $\begin{array}{l}\text { Alto custo. Alta } \\
\text { precisão. } \\
\text { Compatibilidade com } \\
\text { controladores. }\end{array}$ & \\
\hline
\end{tabular}

Fonte: Os autores 
A montagem do protótipo para aquisição de dados ambientais, foi aplicado no monitoramento ambiental de duas casas de vegetação. O monitoramento ambiental consistiu em coletar e armazenar dados de umidade relativa do ar, temperatura do ar, umidade do solo e luminosidade, estes fatores foram escolhidos por serem importantes para o desenvolvimento das culturas vegetais e bastante comuns na pesquisa agropecuária.

Os sensores, da mesma forma que o sistema de controle deveriam seguir a premissa do baixo custo, assim, para monitorar umidade e temperatura atmosférica, optou-se pelo termo-higrômetro modelo DHT-22, devido sua compatibilidade com o sistema Arduino e baixo percentual de erro médio na aquisição, de $\pm 0,5^{\circ} \mathrm{C}$ para temperatura e $\pm 2 \%$ para umidade relativa, fato que, em condições agrícolas, atende a maioria das aplicações de pesquisa e controle ambiental.

Para monitorar a luminosidade, foi montado um transdutor do tipo LDR (Light diode resistor), embora com pouca precisão, por se tratar de um sensor resistivo adaptado, o fator determinante para a utilização deste sensor foi o baixo custo. Dessa forma, o sensor de luminosidade apresentava valores aproximados da presença e intensidade de luz no ambiente. O sensor LDR foi calibrado em câmera escura (caixa) para apresentar o valor de $0 \%$ de luminosidade e em sol pleno para $99 \%$ de luminosidade, com ajuste e monitoramento da conversão de tensão em percentual feitas pelo controlador Arduino.

Para monitorar a umidade do solo, optou-se por utilizar o sensor resistivo de umidade do solo modelo FC-28, composto por duas hastes metálicas, recobertas com liga metálica em prata e conversor de sinal eletrônico. O sensor FC-28 monitorou a umidade do solo através da variação da resistência elétrica entre as hastes metálicas ou eletrodos e foi calibrado localmente a exemplo do sensor LDR, com $100 \%$ para o solo saturado com água e para $0 \%$ em uma amostra de solo seca em estufa a $105^{\circ} \mathrm{C}$ por 24 horas.

Uma das premissas do projeto de aquisição de dados, foi coletar as informações ambientais o mais próximo da cultura vegetal, obtendo assim, informações mais fidedignas do microambiente ao qual as plantas estão inseridas e minimizando efeitos de correntes convectivas de ar que poderiam afetar a qualidade dos dados.

Para que os sensores ficassem mais próximos do estrato vegetal, estes foram instalados em suportes de Policloreto de Vinila (PVC), desenvolvidos exclusivamente para o projeto do Datalogger, com $40 \mathrm{~mm}$ de diâmetro e $800 \mathrm{~mm}$ de altura. Para construção dos 
Plataforma arduino aplicada no desenvolvimento de um sistema...

suportes, utilizou-se barras de PVC comercial, disponível em lojas especializadas em instalações hidrossanitárias.

Em cada suporte de PVC foram instalados 03 sensores, sendo eles, 01 DTH-22, 01 LDR e 01 FC-28. As informações dos sensores foram coletadas a cada 5 minutos com o intuito de registrar detalhadamente a variação ambiental ao longo de 24 horas. Na Tabela 1 são apresentadas as características técnicas de cada sensor utilizado para aquisição de dados no sistema.

Tabela 1: Características técnicas dos sensores com base em dados dos fabricantes

\begin{tabular}{|l|l|l|l|}
\hline Tipo de Sensor & DHT22 & FC28 & LDR \\
\hline Tensão de funcionamento (VDC) & $3,0-5,0$ & $2,5-5,0$ & $0-5,0$ \\
\hline Erro médio aquisição $\pm(\%)$ & $\begin{array}{l}0,5^{\circ} \mathrm{C}(\text { Temp.) } \\
2,0 \%(\mathrm{U} . \mathrm{R})\end{array}$ & 10 & 12 \\
\hline Dimensões (cm) & $2,5 \times 1,5 \times 0,7$ & $5,9 \times 2,0 \times 0,1$ & $0,5 \times 0,55$ \\
\hline Comprimento do cabo (m) & 20 & 20 & 20 \\
\hline Tempo de resposta (s) & $2,0-5,0$ & Imediato & Imediato \\
\hline Faixa de medição (\%) & $0-100$ UR & $0-100$ & $0-100$ \\
\hline Faixa de medição Temperatura & -40 a $80^{\circ} \mathrm{C}$ & N.A & N.A \\
\hline Garantia & 90 dias & Sem Gar. & N.A \\
\hline
\end{tabular}

Fonte: Os autores

O sistema de coleta de dados foi instalado para monitorar o ambiente interno de duas casas de vegetação modelo túnel alto, com $50 \mathrm{~m}^{2}$ cada (Figura 2). Os ambientes foram identificados como $A 1$ e $A 2$, sendo que no ambiente $A 1$ foram instalados os mecanismos de controle ambiental de ventilação, nebulização e irrigação automáticas. Na casa de vegetação A2 o manejo da irrigação era manual e o ambiente não possuía nenhum mecanismo de atenuação ambiental como ventiladores e nebulizadores. 
Figura 2: Esquema de montagem do Arduino Datalogger montado em casas de vegetação $A 1$ e A2 para coleta de dados microambientais no estrato da cultura vegetal.

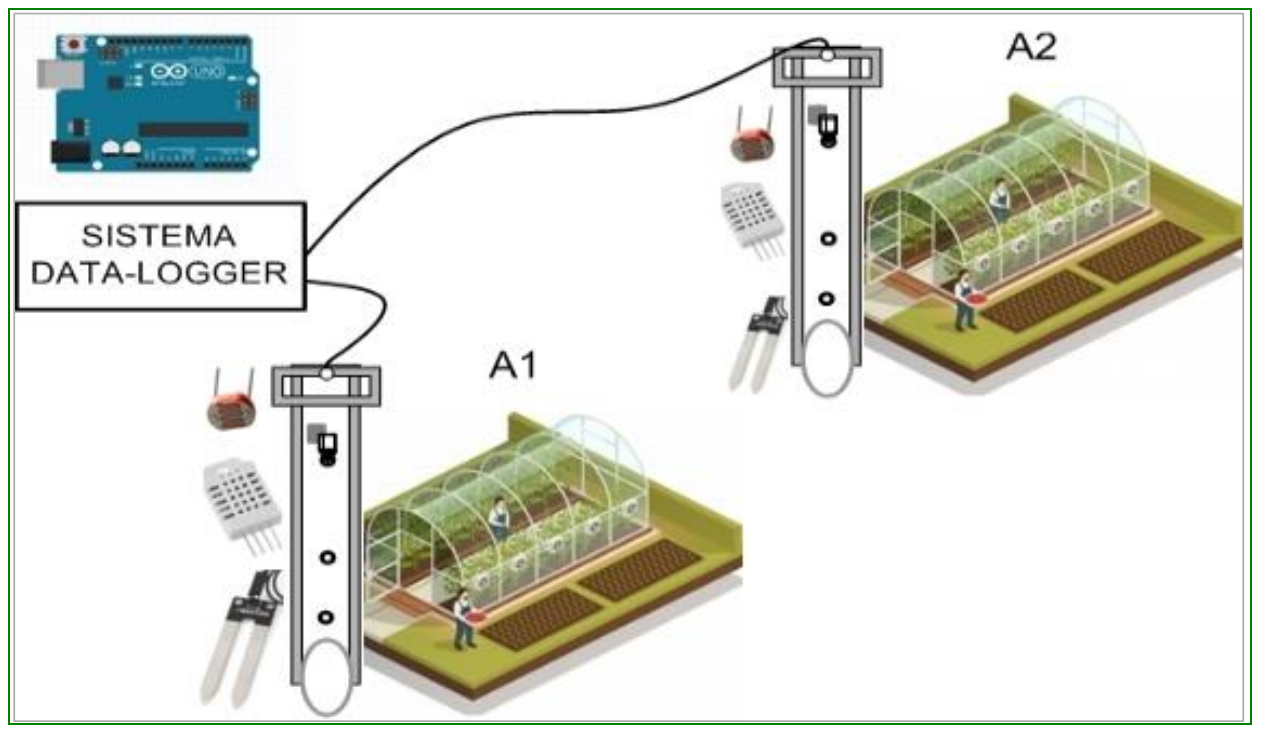

Fonte: Os autores

O sistema de controle ambiental, integrado em A1 e A2, foi avaliado por Marques Filho et al. 2020. Os autores verificaram o incremento na produtividade da alface através da tecnologia de controle com a plataforma Arduino.

Para a avaliação do sistema, o Datalogger foi programado para realizar aquisições e leitura dos sensores em intervalos de 5 minutos, de forma que, os resultados de cada hora de funcionamento foram obtidos através da média simples de 12 aquisições ao longo de 60 minutos.

As casas de vegetação foram construídas de forma paralela, onde as conexões entre o dispositivo Datalogger (Figura 3 c) e as torres de aquisição de dados (Figura 3 a) foram realizadas com cabo do tipo manga ( $8 \times 18 \mathrm{AWG})$, com blindagem e proteção de malha externa em cobre (Figura 3 a). $\mathrm{O}$ dispositivo eletrônico de coleta de dados, bem como 0 sistema de fornecimento de energia, fontes e conexões foram dispostos em local abrigado da chuva e do sol entre as duas casas de vegetação (Figura 3 b). 
Figura 3: Montagem e instalação do sistema Arduino Datalogger em duas casas de vegetação para coleta de dados microambientais no estrato da cultura vegetal.

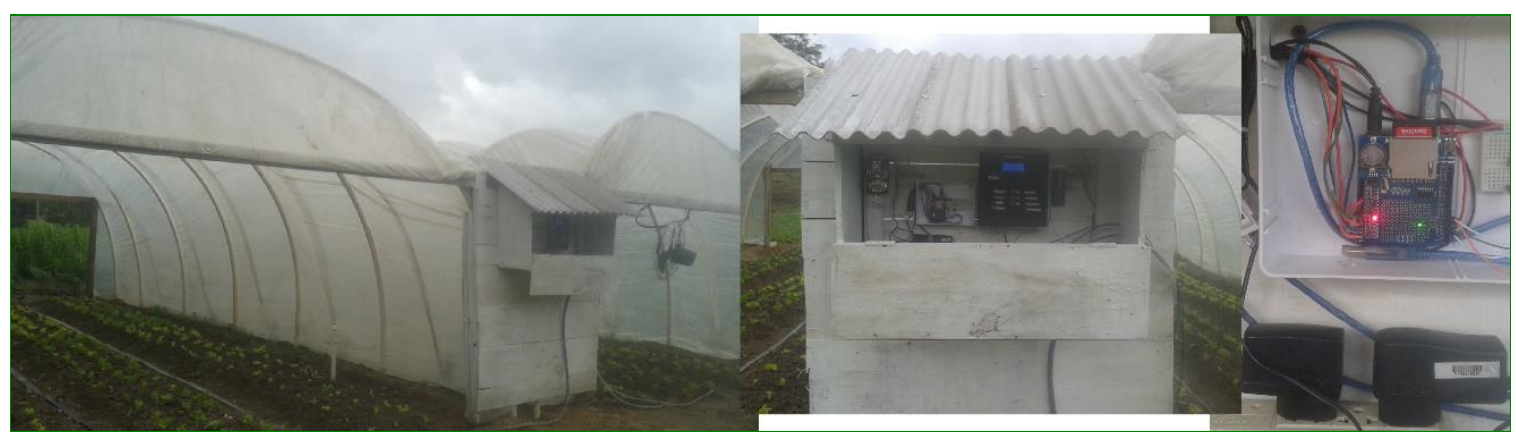

Fonte: Os autores

Todos os dados obtidos pelos sensores foram armazenados automaticamente, através de programação prévia, em arquivo digital do cartão SD, em extensão .LOG e, posteriormente o cartão foi retirado ao final do período experimental e inserido em computador portátil, onde os arquivos originais foram convertidos em formato .XML no software Microsoft Excel 365.

A conversão dos dados em .XML ocorreu para facilitar a edição e criação de planilhas eletrônicas de dados e gráficos ambientais, onde foram representadas a temperatura $\left(\mathrm{C}^{\circ}\right)$, a umidade relativa do ar (\%), a luminosidade (\%) e a umidade do solo (\%) ao longo do período experimental.

Os resultados obtidos no trabalho foram interpretados e discutidos com base em estatística descritiva, em que, realizou-se através do sistema de coleta e aquisição de dados, uma análise do comportamento das variáveis nos ambientes A1 e A2. Realizou-se ainda, uma pesquisa básica de mercado referente aos custos de sistemas comerciais tradicionais que fariam a mesma função do Datalogger desenvolvido, efetuando-se o comparativo de preços entre as diversas tecnologias.

\section{RESULTADOS E DISCUSSÃO}

O dispositivo desenvolvido permitiu a aquisição de dados nos ambientes A1 e A2 ao longo de 24 horas de coleta de dados, sem interrupções ou falhas. No decorrer do período de aquisição de dados, não foram verificados problemas de funcionamento no sistema de aquisição nem inconstância de sinal dos sensores. A Figura 4 demonstra o comportamento das variáveis ambientais na casa de vegetação A1 ao longo do período experimental de aquisição de dados com o dispositivo Datalogger de baixo custo. 
Figura 4: Dispositivo de coleta de dados Arduino Datalogger montado no ambiente A1 em monitoramento das variáveis de umidade do solo, luminosidade, umidade do ar e temperatura ao longo de 24 horas

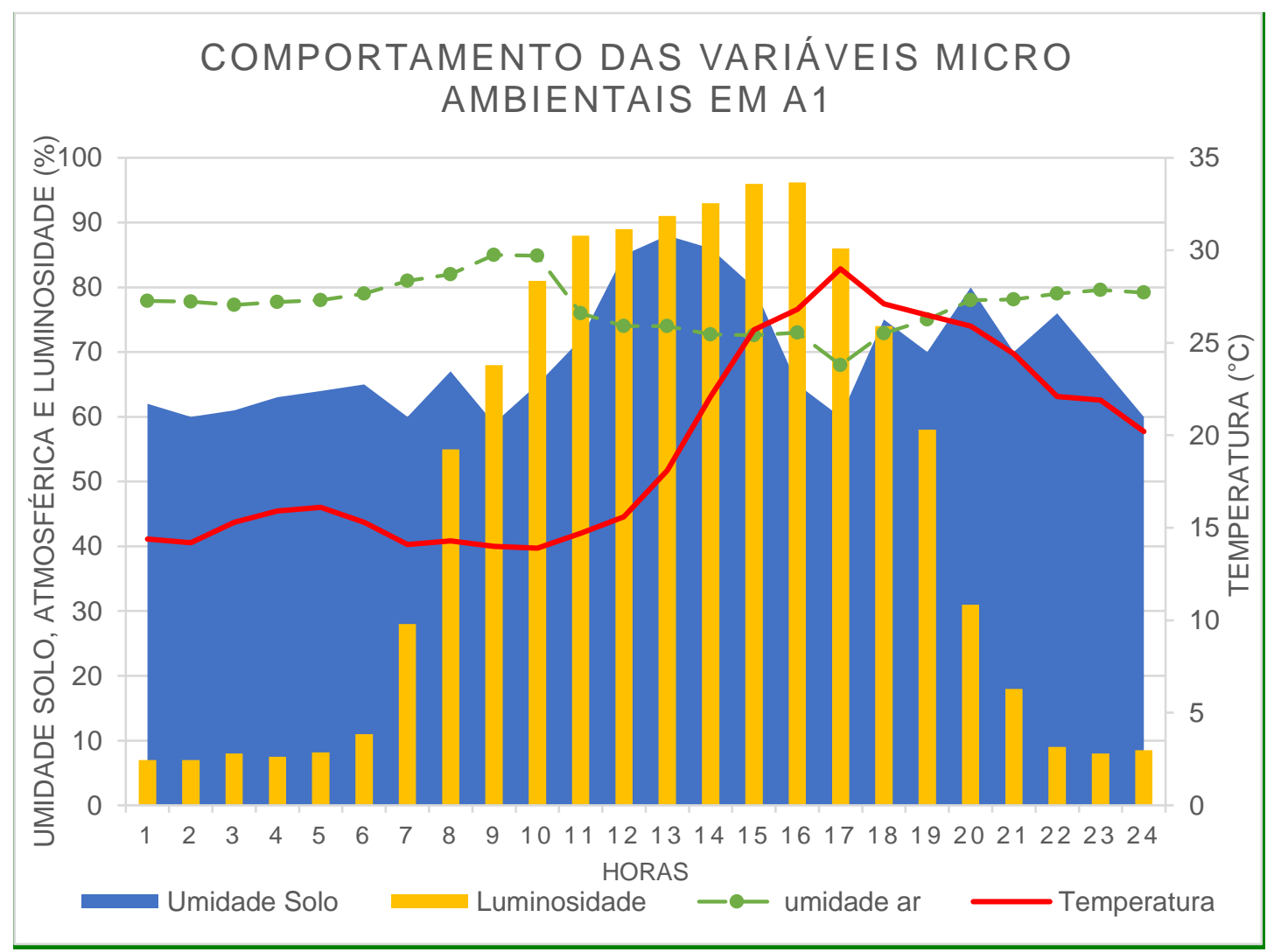

Fonte: Os autores

A obtenção dos dados obtidos pelo sistema Datalogger, permitiu a representação ambiental em A1 para as variáveis umidade do solo, luminosidade, umidade do ar e temperatura. Verificou-se o aumento da luminosidade a partir das $6 \mathrm{hs}$ da manhã, atingindo seu pico entre 15 e 16 horas, o que indica que, mesmo sem ter a alta precisão unitária da radiação, como em um piranômetro por exemplo, o sensor de luminosidade representa boa sensibilidade à luminosidade global. É possível que o sensor LDR possa ser melhor calibrado e comparado a algum equipamento comercial preciso, entretanto, essa avaliação não foi realizada nesta pesquisa. Para aplicações onde a luminosidade não exige alta precisão, como no caso de controle de luminosidade em aviários, monitoramento do dia e da noite, fotoperíodo, entre outros, o LDR apresenta aplicabilidade bastante eficaz. 
A temperatura ambiente apresentou relação positiva com a luminosidade e inversa à umidade relativa do ar, fato esperado para essas variáveis de acordo com a relação dos parâmetros ambientais em casas de vegetação preconizados por Teruel (2010).

Em A1 a umidade do solo manteve-se dentro dos parâmetros de controle, entre 60 e $80 \%$, ao longo do período experimental, fato que pode ser explicado pelo fato da casa de vegetação possuir controle automatizado de irrigação. Ali et al. (2016), em pesquisa sobre o desenvolvimento de um sistema de coleta de dados ambientais com a plataforma Arduino UNO, equipado com o microcontrolador Atmega 328P, obtiveram resultados satisfatórios de funcionamento e durabilidade do sistema, corroborando com os resultados obtidos nesta pesquisa.

A gravação dos dados em arquivo formato “.LOG” permitiu gerar uma quantidade substancial de informações meteorológicas ocupando pouco espaço na memória do cartão SD. As coletas, realizadas a cada 05 minutos ao longo de 24 horas, geraram um arquivo com o tamanho total de 2,44MB. Evidentemente, a densidade de coleta de dados e seu intervalo de gravação podem ser reduzidos, já que a modificação dos parâmetros ambientais sofre baixa alteração significativa em curtos intervalos de tempo.

Normalmente os agricultores e pesquisadores necessitam de longos períodos de tempo para monitoramento e aquisição de dados, portanto, os resultados obtidos nesta pesquisa carecem ainda de maior período experimental em diferentes validações, tais como, testar o sistema de aquisição em outras condições ambientais e realizar comparativos com as tecnologias existentes. Entretanto, o Datalogger de baixo custo mostrou-se promissor, mesmo necessitando de aprimoramentos nos sensores de luminosidade e umidade do solo, os quais devem ser dimensionados de acordo com a criticidade de aplicação do projeto e necessidade de precisão nos dados.

No ambiente A2 (Figura 5), observa-se como principal diferença em relação ao ambiente $A 1$, a desuniformidade da umidade do solo. Os parâmetros de temperatura, umidade relativa do ar e radiação sofreram pouca diferença, embora com menores temperaturas e maiores umidades em relação à casa de vegetação $A 1$. Este comportamento foi explicado por Marques Filho et al. (2020), devido à ineficiência dos dispositivos atuadores no controle das variáveis microclimáticas neste ambiente, de forma que somente os atuadores de irrigação mantiveram o processo sob controle. Entretanto, percebe-se que o ambiente $A 1$, mesmo com a baixa eficiência de controle ambiental, teve 
atenuação de temperatura e umidade relativa em relação ao ambiente $A 2$, fato que ficou evidenciado pelos dados obtidos no sistema Datalogger desenvolvido nesta pesquisa.

Figura 5: Dispositivo de coleta de dados Arduino Datalogger montado no ambiente A2 em monitoramento das variáveis de umidade do solo, luminosidade, umidade do ar e temperatura ao longo de 24 horas.

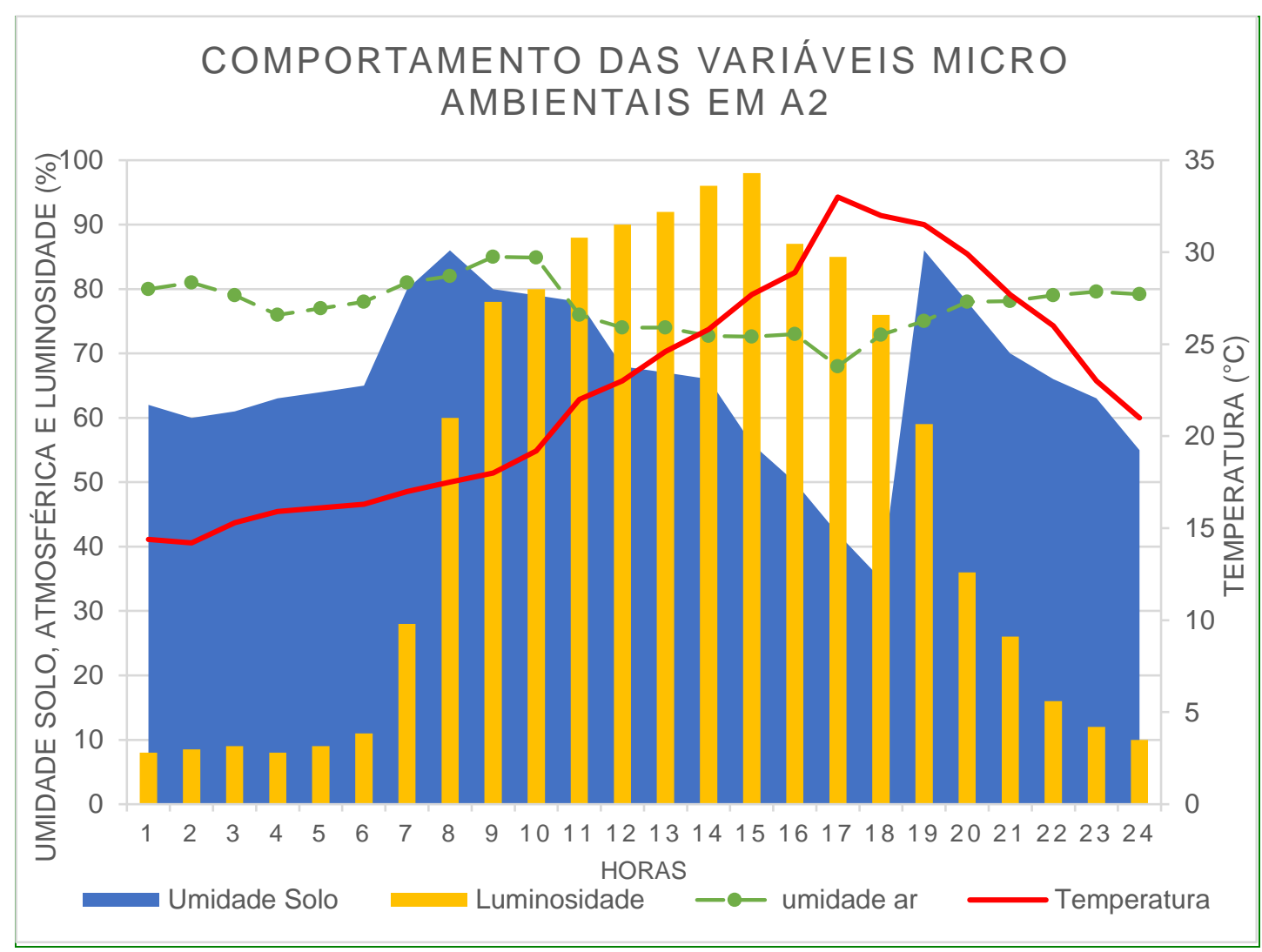

Fonte: Os autores

O custo para montagem do sistema de coleta de dados, incluindo os suportes de sensores em PVC e dispositivos acessórios está descrito na Tabela 2. Estes valores são resultados de pesquisas de mercado realizadas entre os dias 01/04/2021 e 01/06/2021 com valores atualizados dos dispositivos em lojas do mercado nacional e convertidos em dólares americanos (Valor do dólar: $\mathrm{R} \$ 5,04$ - em 06/2021). 
Plataforma arduino aplicada no desenvolvimento de um sistema...

Tabela 2: Custos de equipamentos e construção de um Datalogger com dois suportes de sensores para aquisição de dados ambientais

\begin{tabular}{|c|c|l|l|l|}
\hline Material & Quantidade & Unidade & Valor & Total \\
\hline Gabinete Plástico 15x20cm & 1 & $\mathrm{pC}$ & $\mathrm{U} \$ 2,90$ & $\mathrm{U} \$ 2,90$ \\
\hline Placa Arduino UNO & 1 & $\mathrm{pc}$ & $\mathrm{U} \$ 13,69$ & $\mathrm{U} \$ 13,69$ \\
\hline SD Shield + RTC I2C +SD2GB & 1 & $\mathrm{pC}$ & $\mathrm{U} \$ 11,90$ & $\mathrm{U} \$ 11,90$ \\
\hline Resistor 10KOhm & 4 & $\mathrm{pC}$ & $\mathrm{U} \$ 0,01$ & $\mathrm{U} \$ 0,04$ \\
\hline SENSOR Umidade e Temp DHT22 & 2 & $\mathrm{pc}$ & $\mathrm{U} \$ 6,74$ & $\mathrm{U} \$ 13,48$ \\
\hline Sensor Umidade de solo FC-28 & 2 & $\mathrm{pc}$ & $\mathrm{U} \$ 3,75$ & $\mathrm{U} \$ 7,50$ \\
\hline Resistor LDR & 2 & $\mathrm{pc}$ & $\mathrm{U} \$ 0,25$ & $\mathrm{U} \$ 0,50$ \\
\hline PVC 40mm & 2 & $\mathrm{~m}$ & $\mathrm{U} \$ 0,90$ & $\mathrm{U} \$ 1,80$ \\
\hline Tampa PVC 40mm & 2 & $\mathrm{pc}$ & $\mathrm{U} \$ 0,28$ & $\mathrm{U} \$ 0,56$ \\
\hline Fonte 9V (DC) .1 a & 1 & $\mathrm{pc}$ & $\mathrm{U} \$ 2,90$ & $\mathrm{U} \$ 2,90$ \\
\hline Conexões cabos e montagem & 1 & $\mathrm{vb}$ & $\mathrm{U} \$ 50,00$ & $\mathrm{U} \$ 50,00$ \\
\hline \hline Total & & & & $\mathrm{U} \$ 105,27$ \\
\hline
\end{tabular}

Fonte: Os autores

O custo de dispositivos Dataloggers comerciais com funcionalidade semelhante ao desenvolvido neste estudo, em média, ficam acima de 700 dólares americanos no mercado nacional, excetuando-se ainda os sensores. Foram realizados orçamentos de diversos sistemas de aquisição de dados em sites de fabricantes distintos, com fabricação nacional e importados, os nomes das empresas foram preservados, entretanto, as características e os principais valores obtidos estão descritos na Tabela 3.

Tabela 3: Custos e características técnicas básicas de Dataloggers comerciais

\begin{tabular}{|c|c|c|l|l|}
\hline Sistema Datalogger & Fabricação & $\begin{array}{c}\text { Canais ou } \\
\text { portas I/O } \\
\text { Analógicas }\end{array}$ & $\begin{array}{l}\text { Canais ou } \\
\text { portas I/O } \\
\text { digitais }\end{array}$ & Preço/Custo \\
\hline Fabricante 1 & Nacional & 6 & 10 & $\mathrm{U} \$ 815,4$ \\
\hline Fabricante 2 & Importado & 2 & 4 & $\mathrm{U} \$ 713,2$ \\
\hline Fabricante 3 & Importado & 4 & 8 & $\mathrm{U} \$ 1360,0$ \\
\hline Fabricante 4 & Importado & 12 & 24 & $\mathrm{U} \$ 3570,0$ \\
\hline Protótipo Arduino & Protótipo & 6 & 14 & $\mathrm{U} \$ 105,3$ \\
\hline \hline & & & & \\
\hline
\end{tabular}

Fonte: Os autores

Considerando o valor de desenvolvimento do protótipo Datalogger em comparação com a tecnologia de mercado com menor preço (Fabricante 2 - 713,2 U\$), verifica-se que a tecnologia de mercado apresenta custo $677 \%$ superior ao protótipo desenvolvido. Ao comparar-se com a tecnologia de maior custo (Fabricante 4 - 3570 U\$) encontra-se um 
acréscimo superior a 3300\%. Para Fuentes et al. (2014) é vantajoso o desenvolvimento de Dataloggers de baixo custo utilizando microcontroladores e sistemas eletrônicos simplificados, pois estes apresentam desempenho semelhante aos sistemas comerciais a custos expressivamente mais baixos.

Resultados semelhantes a este trabalho foram obtidos por Lee et al. (2014), em monitoramento ambiental de estações meteorológicas, substituindo o sistema tradicional de aquisição de dados por sistemas e sensores em plataforma Arduino. Baker (2014) afirmou que a utilização das plataformas abertas e softwares livres permitem que usuários sem muita experiência na área da eletrônica e automação, possam desenvolver trabalhos interessantes e aplicáveis em seus meios de atuação, fato que é igualmente ratificado por McRoberts (2018).

Ali et al. (2016) afirmaram que o desenvolvimento de um sistema integrado, composto por componentes oriundos de distintos fabricantes, pode apresentar problemas de funcionamento. Os autores obtiveram dificuldades com a integração de bibliotecas e códigos específicos, ponderando que, o baixo custo em equipamentos e montagens pode ser afetado quando se leva em consideração as fontes de erro que o sistema pode oferecer e o tempo que o mesmo toma para ser construído. Nesta pesquisa e desenvolvimento, não obteve-se problemas de incompatibilidade, fato que pode ser melhor investigado em pesquisas futuras e maiores períodos experimentais.

Martinez-Santos; Acevedo-Patino e Contreras-Ortiz (2017), concluíram que as plataformas de desenvolvimento Arduino contribuem para o aprendizado em ambientes educacionais que envolvem projetos e desenvolvimento de produtos, entretanto, a ampla oferta de equipamentos, sensores e tecnologias disponíveis no mercado, necessitam de seleção rigorosa, pois podem ser fontes de seleção inadequada dos dispositivos para cada projeto.

Na presente pesquisa, o fator seleção de equipamentos foi balizado pelo baixo custo dos equipamentos de coleta de dados, já que o objetivo principal foi desenvolver o sistema de aquisição, entretanto, em aplicações mais sensíveis onde a precisão dos sensores é exigida, devem-se optar por sensores com maior precisão em detrimento ao baixo custo.

Como aprimoramentos futuros ao sistema desenvolvido na presente pesquisa, está a possibilidade da eliminação do cabeamento entre os sensores e o sistema Datalogger, de forma a facilitar a instalação e mobilidade dos suportes de sensores no ambiente agrícola. Iberahim et al. (2017) ratificaram que as utilizações de diversas tecnologias sem 
fio estão mudando a forma como o mundo se relaciona com os dispositivos eletrônicos e abrindo novas oportunidades com frentes de trabalho promissoras.

Mabrouki et al. (2021) realizaram o monitoramento ambiental utilizando tecnologias sem fio com excelentes resultados e baixo custo. Os dispositivos eletrônicos para estas aplicações tornaram-se mais acessíveis e com menor preço, o que possibilita a aplicação em diversos projetos e áreas de atuação. Tecnologias de transmissão sem fio, como redes de longo alcance e baixo consumo de energia em sistemas LPWAN e LORA seriam perfeitamente aplicáveis no presente projeto, e ficam como referências para aprimoramentos futuros e novas pesquisas.

\section{CONCLUSÕES}

A plataforma de prototipagem Arduino, em conjunto com sensores de baixo custo, podem ser perfeitamente aplicáveis para a aquisição e armazenamento de dados no meio agrícola, sendo que a precisão dos sensores varia de acordo com a necessidade de aplicação da tecnologia.

O protótipo de Datalogger desenvolvido neste estudo, atendeu às expectativas iniciais do projeto em relação ao custo total, apresentou uma redução de custo de 600 até $3000 \%$ quando comparado com tecnologias semelhantes disponíveis no mercado.

\section{REFERÊNCIAS}

AGUDO, F. L.; SANTINI, G. A.; PIGATTO S.; GOBBO JÚNIOR, J. A.; BAPTISTA, R. D.; QUEIROZ, T. R. Aplicação da estratégia open innovation na agricultura mundial: contribuições a partir de revisão sistematizada da literatura. Brazilian Journal of Biosystems Engineering, v.14, n.3, p.208-231, 2020.

ALI, A. S.; ZANZINGER, Z.; DEBOSE, D.; STEPHENS, B. Open Source Building Science Sensors (OSBSS): A low-cost Arduino based platform for long-term indoor environmental data collection. Building and Environment, Chicago, IL., v.100, p.114-126, 2016.

BAKER, E. Open source data logger for low-cost environmental monitoring. Biodiversity Data Journal, London v.2, ed.1059, 2014.

BENGHANEM, M. Measurement of meteorological data based on wireless data acquisition system monitoring. Applied Energy, Madinah, A.S. v.86, p.2651-2660, 2009.

FUENTES, M.; VIVAR, M.; BURGOS, J.M.; AGUILERA, J.; VACAS, J. A. Design of an accurate, low-cost autonomous data logger for PV system monitoring using Arduino ${ }^{\mathrm{TM}}$ that complies with IEC standards. Solar Energy materials and Solar cells, v.130, p.529-543, 2014. 
GOPINATH, A.; ARUN, C.; HANUMANTHAIAH, A.; MURUGAN, R. An Analogy of the Datalogger Implementation in Arduino UNO and PSoC5LP. In 2020 Third International Conference on Smart Systems and Inventive Technology (ICSSIT), IEEE, pp. 328-332, 2020.

IBERAHIM, H. H.; BASARUDIN, H.; ABU, M. H.; SENG, G. H.; RAMLI, A. F.; SULAIMAN, M. I. Development of Wireless Transmission for Meteorological Stations Data Logging. IEEE, International Conference on Engineering Technology and Technopreneurship (ICE2T), Kuala Lumpur, 2017.

LEE, S.; KIM, Y.; JO, J.; STEPHEN, H. A Framework for Environmental Monitoring with Arduino-based Sensors using Restful Web Service. IEEE, International Conference on Services Computing. Nevada, 2014.

MABROUKI, J.; AZROUR, M.; DHIBA, D.; FARHAOUI, Y.; EL HAJJAJI, S. IoT-based data logger for weather monitoring using arduino-based wireless sensor networks with remote graphical application and alerts. Big Data Mining and Analytics, v. 4, n. 1, p. 25-32, 2021.

MARQUES FILHO, A. C.; RODRIGUES, J. P.; MEDEIROS, S. D. S.; S. R. R. M. Development of an electronic controller for lettuce production in greenhouses. Revista de Agricultura Neotropical, v. 7, n. 3, p.65-72, 2020.

MARTINEZ-SANTOS, J. C.; ACEVEDO-PATINO, O.; CONTRERAS-ORTIZ, S. H. Influence of Arduino on the development of advanced microcontrollers courses. IEEE revista iberoamericana de tecnologias del aprendizaje, v. 12, n. 4, p. 208-217, 2017.

MCROBERTS, M. Arduino básico. Novatec Editora, 2.ed, São Paulo, 2018.

MEGANTORO, P.; WIDJANARKO, A.; RAHIM, R.; KUNAL, K.; ARFIANTO, A. Z. The design of digital liquid density meter based on Arduino. Journal of Robotics and Control, v.1, n.1, p.1-6, 2020.

NÓBREGA, L., GONÇALVES, P., ANTUNES, M., CORUJO, D. Assessing sheep behavior through low-power microcontrollers in smart agriculture scenarios. Computers and Electronics in Agriculture, v.173, 2020.

OLADIMEJI, I.; ADEDIJI, Y. B.; AKINTOLA, J. B.; AFOLAYAN, M. A.; OGUNBIYI, O.; IBRAHIM, S. M.; OLAYINKA, S. Z. Design and construction of an arduino-based solar power parameter-measuring system with data logger. Arid Zone Journal Of Engineering, Technology And Environment, v. 16, n. 2, p. 255-268, 2020.

ROZENFELD, H.; FORCELLINI, F. A.; AMARAL, D. C.; TOLEDO, J. C.; SILVA, S. L.; ALLIPRANDINI, D. H.; SCALICE, R. K. Gestão de desenvolvimento de Produtos. Uma referência para melhoria do processo. São Paulo. Ed. Saraiva, 2015. 542p.

TERUEL, B. J. Controle automatizado de casas de vegetação: Variáveis climáticas e fertigação. Revista Brasileira de Engenharia Agrícola e Ambiental, v. 14, n. 3, p. 237245, 2010. 


\begin{abstract}
Data collection and environmental monitoring in agriculture are highly complex activities, normally requiring expensive equipment. The objective was to develop a low-cost data collection system to obtain parameters related to luminosity, soil moisture, air humidity and temperature in an agricultural environment. The Arduino prototyping platform was used to assemble the Datalogger system. Functional tests were carried out in two greenhouses A1 and A2, for a period of 24 hours. The design of the system, with the use of sensor supports, allowed the collection of data without interruption and with adequate storage in the device's memory. The Arduino platform and accessory sensors proved to be perfectly applicable for data acquisition and storage in greenhouses. The developed Datalogger prototype showed a cost reduction of 600 to $3000 \%$ compared to components available on the market with similar functionalities.
\end{abstract}

Keywords: Research. Sensors. Automation. Precision Agriculture.

\title{
RESUMEN
}

La recopilación de datos y el monitoreo ambiental en la agricultura son actividades muy complejas que requieren equipos costosos. El objetivo fue desarrollar un sistema de recolección de datos de bajo costo para obtener parámetros relacionados con la luminosidad, la humedad del suelo, la humedad del aire y la temperatura en un ambiente agrícola. La plataforma de creación de prototipos Arduino se utilizó para ensamblar el sistema Datalogger. Se realizaron pruebas funcionales en dos invernaderos A1 y A2, por un período de 24 horas. El diseño del sistema, con el uso de soportes de sensores, permitió la recolección de datos sin interrupciones y con un almacenamiento adecuado en la memoria del dispositivo. La plataforma Arduino y los sensores accesorios demostraron ser perfectamente aplicables para la adquisición y almacenamiento de datos en invernaderos. El prototipo de Datalogger desarrollado mostró una reducción de costos de 600 a 3000\% en comparación con los componentes disponibles en el mercado con funcionalidades similares.

Palabras clave: Investigar. Sensores. Automatización. Agricultura de precisión 


\section{LICENÇA DE USO}

Este é um artigo publicado em acesso aberto (Open Access) sob a licença Creative Commons Atribuição 4.0 Internacional (CC BY 4.0), que permite uso, distribuição e reprodução em qualquer meio, desde que o trabalho original seja corretamente citado. Mais informações em: http://creativecommons.org/licenses/by/4.0

\section{CONFLITO DE INTERESSES}

Os autores declaram que não há conflito de interesses neste trabalho.

\section{CONTRIBUIÇÕES AUTORAIS}

Autor 1: Responsável pela autoria de todo o texto.

Autor 2: Responsável coautoria de todo o texto.

Autor 3: Responsável coautoria de todo o texto.

\section{FINANCIAMENTO}

O presente trabalho foi realizado com apoio do CNPQ, projeto PROPPI UNIVERSAL 02/2016, da Pró-Reitoria de Pesquisa, Pós-Graduação e Inovação.

\section{COMO REFERENCIAR}

FILHO, Aldir Carpes Marques; RODRIGUES, Jean Paulo; DAL PONTE, Gabriel Buligon. Plataforma arduino aplicada no desenvolvimento de um sistema de coleta de dados micro-ambientais em casas de vegetação. Revista Brasileira de Engenharia de Biossistemas (Tupã), v. 15, n. 2, p. 190-206, 2021. DOI:

http://dx.doi.org/10.18011/bioeng2021v15n2p190-206.

\section{RESPONSABILIBADE EDITORIAL}

Prof. Dr. Fernando Ferrari Putti ${ }^{1}$, Prof. Dr. Paulo Sérgio Barbosa dos Santos ${ }^{1}$, Prof. Dr. Eduardo Festozo Vicente ${ }^{1}$ e Prof. Dr. Diogo de Lucca Sartori ${ }^{1}$

${ }^{1}$ Universidade Estadual Paulista "Júlio de Mesquita Filho", FCE - Faculdade de Ciências e Engenharia, Tupã, SP, Brasil. 